\title{
Assessment of the direct effects of DDAH I on tumour angiogenesis in vivo
}

\author{
Efthymia Papaevangelou ${ }^{1,3}$ (1) Jessica K. R. Boult ${ }^{2} \cdot$ Guy S. Whitley $^{1} \cdot$ Simon P. Robinson $^{2} \cdot$ Franklyn A. Howe $^{1}$
}

Received: 24 August 2017 / Accepted: 24 April 2018 / Published online: 2 May 2018

(c) The Author(s) 2018

\begin{abstract}
Nitric oxide (NO) has been strongly implicated in glioma progression and angiogenesis. The endogenous inhibitors of NO synthesis, asymmetric dimethylarginine (ADMA) and $N$-monomethyl-L-arginine (L-NMMA), are metabolized by dimethylarginine dimethylaminohydrolase (DDAH), and hence, DDAH is an intracellular factor that regulates NO. However, DDAH may also have an NO-independent action. We aimed to investigate whether DDAH I has any direct role in tumour vascular development and growth independent of its NO-mediated effects, in order to establish the future potential of DDAH inhibition as an anti-angiogenic treatment strategy. A clone of rat C6 glioma cells deficient in NO production expressing a pTet Off regulatable element was identified and engineered to overexpress DDAH I in the absence of doxycycline. Xenografts derived from these cells were propagated in the presence or absence of doxycycline and susceptibility magnetic resonance imaging used to assess functional vasculature in vivo. Pathological correlates of tumour vascular density, maturation and function were also sought. In the absence of doxycycline, tumours exhibited high DDAH I expression and activity, which was suppressed in its presence. However, overexpression of DDAH I had no measurable effect on tumour growth, vessel density, function or maturation. These data suggest that in C6 gliomas DDAH has no NO-independent effects on tumour growth and angiogenesis, and that the therapeutic potential of targeting DDAH in gliomas should only be considered in the context of NO regulation.
\end{abstract}

Keywords DDAH $\cdot$ Nitric oxide $\cdot$ Inducible expression $\cdot$ MRI $\cdot$ Tumour angiogenesis

\section{Introduction}

Angiogenesis is an important driver of the growth and development of solid tumours. Several angiogenic stimuli also induce the production of nitric oxide (NO). Strong positive correlations between the expression of nitric oxide synthases

Electronic supplementary material The online version of this article (https://doi.org/10.1007/s10456-018-9617-6) contains supplementary material, which is available to authorized users.

Efthymia Papaevangelou

efthymia.papaevangelou@kcl.ac.uk

1 Molecular and Clinical Sciences Research Institute, St. George's, University of London, Cranmer Terrace, London SW17 ORE, UK

2 Division of Radiotherapy and Imaging, The Institute of Cancer Research, 15 Cotswold Road, Sutton, Surrey SM2 5NG, UK

3 School of Immunology and Microbial Sciences, Guy's Hospital, King's College London, London, UK
(NOS) and tumour progression have been identified in a number of human cancers [1]. Intracellular factors which regulate NO synthesis may therefore represent important targets in the control of tumour growth and vascularization.

The synthesis of NO can be modulated by the two dimethylarginine dimethylaminohydrolase (DDAH) isoforms via the asymmetric dimethylarginine (ADMA) pathway [2]. DDAH enzymes catalyse the hydrolysis of methylarginines, ADMA and $N$-monomethyl-L-arginine (L-NMMA), to citrulline and either dimethylamine or methylamine, respectively [3]. ADMA and L-NMMA are endogenous, competitive inhibitors of all three NOS isoforms, and NO production is physiologically regulated by methylarginines [4].

DDAH activity has been detected in various human tumours including those arising in the brain; astrocytomas in particular exhibited high levels of DDAH activity [5]. High expression of DDAH I has also been observed in aggressive triple-negative breast cancer cell lines, where DDAH I was a key regulator of cell migration and vasculogenic mimicry [6]. Overexpression of DDAH I has been shown 
to increase NO production and enhance tumour growth and angiogenesis in C6 gliomas in vivo [7, 8]. Pharmacological manipulation of DDAH may be an attractive therapeutic target for regulating NO in cancer. DDAH inhibitors can help overcome one of the major considerations for NO-focused therapies, which is targeting pathological NO production while sparing beneficial NO produced constitutively. Direct non-selective NOS inhibitors can lead to inherent adverse effects by limiting the NO antimicrobial properties or by inhibiting the constitutive NOS isoforms [9].

Previous studies have looked into DDAH-mediated NOdependent effects on tumour vascularization. However, other studies have also suggested that DDAH and/or ADMA can exert direct, NO-independent effects on angiogenesis. For example, DDAH II overexpression led to increased vascular endothelial growth factor (VEGF) expression in endothelial cells by binding to protein kinase A (PKA), increasing phosphorylation and activation of the transcription factor Sp1 [10, 11]. These studies imply that the DDAH/ADMA pathway may directly regulate tumour angiogenesis. We can hypothesize that if DDAH has NO-independent effects, then it might be a better anti-cancer target than NO synthases, as NO operates in a bimodal fashion in cancer and can be both pro- and anti-tumorigenic, depending on its concentration and the tumour microenvironment $[12,13]$.

To investigate whether there are any NO-independent effects of tumour-derived DDAH I on tumour angiogenesis, we first identified a C6-derived glioma cell line deficient in NO production, and genetically modified it to overexpress DDAH I under doxycycline (DOX) regulation. These cells were then characterized and compared against parental and wild-type cells in vitro. Subsequently, the vascular phenotype of tumours derived from the regulatable cells was compared in the presence or absence of DOX and with tumours derived from constitutive DDAH I overexpressing NO-producing C6 cells. The vascular functionality of these tumours was interrogated in vivo using quantitative noninvasive MRI, and ex vivo histopathological analysis.

\section{Materials and methods}

\section{Cell culture}

Both wild-type and transfected C6 rat glioma cells (European Collection of Cell Cultures, Salisbury, UK) were maintained in Nutrient Ham's F-10 (Sigma-Aldrich, Dorset, UK) medium containing $2 \mathrm{mM}$ L-glutamine, $100 \mathrm{U} / \mathrm{ml}$ penicillin, $0.2 \mathrm{mg} / \mathrm{ml}$ streptomycin and $10 \%(\mathrm{v} / \mathrm{v})$ tetracycline-free fetal bovine serum (Clontech, Saint-Germain-en-Laye, France).

To achieve inducible overexpression of DDAH I, C6 wild-type (wt) cells were engineered to include a DDAH I cDNA sequence combined with a tetracycline-inducible system (pTet Off), which allowed gene expression to be controlled by tetracycline, or as in our study, by its water soluble derivative, DOX. Briefly, C6 cells transfected with the pRevTet-Off-IN vector (Clontech), referred to as C6 parental (par) cells, were kindly provided by Prof. M. Neeman (Weizmann Institute of Science, Rehovot, Israel). These cells were selected for this study as they did not express iNOS and produced limited NO. Full length rat DDAH I cDNA (previously generated by Kostourou et al. [5]) was cloned into the BamHI site of the pTRE2hyg plasmid (Clontech) to generate pTRE2hyg.DDAHI. C6 par cells were transfected with the pTRE2hyg.DDAHI vector using Fugene 6 and selection was performed with $500 \mu \mathrm{g} / \mathrm{ml}$ hygromycin B in the culture medium in the presence of $1 \mu \mathrm{g} / \mathrm{ml}$ DOX. The stably transfected clone (designated C6 DDAH) with the highest DDAH I overexpression in the absence of DOX was chosen for further experimentation, and grown in the presence or absence of $2 \mu \mathrm{g} / \mathrm{ml}$ DOX. Table 1 lists all the cell lines and tumour types used in the present study and their main characteristics.

\section{Nitric oxide production}

Cells were stimulated with cytokines $(10 \mathrm{ng} / \mathrm{ml} \mathrm{TNF}-\alpha$, $1000 \mathrm{U} / \mathrm{ml} \mathrm{IFN}-\gamma)$ and $5 \mu \mathrm{g} / \mathrm{ml}$ LPS. Nitrite and nitrate production provided a proxy measure of NO synthesis. Nitrate was reduced to nitrite using vanadium chloride, and the total nitrate plus nitrite (NOx) determined using the Griess reaction $[14,15]$. All experiments were performed in triplicate.

\section{Western blot analysis}

The protein concentration of cell lysates and tumour homogenates was determined using the Bradford assay [16] and equal amounts of protein were analysed by SDS-PAGE. DDAH I and iNOS expression were detected using goat polyclonal anti-mouse/rat DDAH I antibodies and rabbit polyclonal anti-mouse/rat iNOS antibodies (M-19; sc-650, Santa Cruz, Heidelberg, Germany) respectively. Polyclonal anti- $\alpha$-actin antibodies (Sigma-Aldrich) or polyclonal anti$\alpha$-tubulin antibodies (Sigma-Aldrich) were used to verify equal protein loading. The integrated density of the individual bands for iNOS and DDAH I was measured and corrected using the integrated density of the individual bands for either $\alpha$-actin or $\alpha$-tubulin using ImageJ software [17].

\section{DDAH activity assay}

DDAH I activity was determined by measuring the conversion of ADMA to L-citrulline [18]. Lyophilized cells (200 $\mu \mathrm{g}$ protein) were mixed with $20 \mu \mathrm{l} 4 \mathrm{mM}$ ADMA in phosphatebuffered saline (PBS, pH 6.5) and incubated for $4 \mathrm{~h}$ at $37^{\circ} \mathrm{C}$. The enzymatic reaction was stopped using an equal volume 
Table 1 List of cell lines and tumours used in the present study and their main characteristics

\begin{tabular}{|c|c|c|c|c|c|c|c|}
\hline \multicolumn{8}{|l|}{ In vitro experiments } \\
\hline Cell line name & \multicolumn{2}{|l|}{ C6 wt } & \multicolumn{2}{|l|}{ C6 parental } & \multicolumn{3}{|c|}{ C6 DDAH } \\
\hline Description & \multicolumn{2}{|l|}{ Wild-type C6 cells } & \multicolumn{2}{|c|}{$\begin{array}{l}\text { C6 cells with pTet-Off } \\
\text { element (parental to C6 } \\
\text { DDAH cells) }\end{array}$} & \multicolumn{3}{|c|}{ DOX-inducible DDAH I overexpressing C6 cells } \\
\hline DOX & \multicolumn{2}{|l|}{ Independent } & \multicolumn{2}{|l|}{ Independent } & \multicolumn{2}{|c|}{ Absence (-DOX) } & Presence (+DOX) \\
\hline $\begin{array}{l}\text { DDAH I expres- } \\
\text { sion }\end{array}$ & \multicolumn{2}{|c|}{ Low endogenous expression } & \multicolumn{2}{|c|}{ Low endogenous expression } & \multicolumn{2}{|c|}{ Overexpression } & Low endogenous expression \\
\hline NO production & \multicolumn{2}{|l|}{ Increased by cytokines } & \multicolumn{2}{|c|}{$\begin{array}{l}\text { Negligible (cytokine inde- } \\
\text { pendent) }\end{array}$} & \multicolumn{2}{|c|}{$\begin{array}{l}\text { Negligible (cytokine inde- } \\
\text { pendent) }\end{array}$} & $\begin{array}{l}\text { Negligible (cytokine inde- } \\
\text { pendent) }\end{array}$ \\
\hline VEGF expression & \multicolumn{2}{|l|}{ Endogenous } & Endogenous & & \multicolumn{2}{|l|}{ Low } & Low \\
\hline \multicolumn{8}{|l|}{ In vivo experiments } \\
\hline Tumour type & \multicolumn{3}{|c|}{$\begin{array}{l}\text { C6 DDAH group A (from cells grown in normal } \\
\text { medium) }\end{array}$} & \multicolumn{3}{|c|}{$\begin{array}{l}\text { C6 DDAH group B (from cells grown in } \\
\text { medium with DOX) }\end{array}$} & D27 \\
\hline Description & \multicolumn{3}{|c|}{$\begin{array}{l}\text { DOX-inducible DDAH I overexpressing C6 } \\
\text { cells }\end{array}$} & \multicolumn{3}{|c|}{$\begin{array}{l}\text { DOX-inducible DDAH I overexpressing C6 } \\
\text { cells }\end{array}$} & $\begin{array}{l}\text { Constitutively DDAH } \\
\text { I overexpressing C6 } \\
\text { cells }\end{array}$ \\
\hline DOX & Absence (-DOX) & \multicolumn{2}{|c|}{ Presence $(+\mathrm{DOX})$} & \multicolumn{2}{|c|}{ Absence (-DOX) } & Presence $(+\mathrm{DOX})$ & Independent \\
\hline $\begin{array}{l}\text { DDAH I expres- } \\
\text { sion }\end{array}$ & Overexpression & \multicolumn{2}{|c|}{$\begin{array}{l}\text { Low endogenous } \\
\text { expression }\end{array}$} & \multicolumn{2}{|c|}{ Overexpression } & $\begin{array}{l}\text { Low endogenous } \\
\text { expression }\end{array}$ & Overexpression \\
\hline NO production & Negligible & \multicolumn{2}{|c|}{ Negligible } & \multicolumn{2}{|c|}{ Negligible } & Negligible & High \\
\hline VEGF expression & Low & \multicolumn{2}{|l|}{ Low } & \multicolumn{2}{|l|}{ Low } & Low & Very high \\
\hline
\end{tabular}

of $10 \%(\mathrm{w} / \mathrm{v})$ trichloroacetic acid. Samples were centrifuged and the supernatants were mixed with a 2:1 ratio mixture of antipyrine $(0.5 \%(\mathrm{w} / \mathrm{v})$ in $50 \%$ sulphuric acid) and diacetyl monoxime $(0.8 \%(\mathrm{w} / \mathrm{v})$ in $5 \%$ acetic acid). Subsequently, samples were heated in a boiling water bath for $30 \mathrm{~min}$ and the amount of L-citrulline formed was determined by measuring the absorbance at $466 \mathrm{~nm}$. To detect DDAH I activity in tumours, tumour homogenates ( $400 \mu \mathrm{g}$ of protein) were initially incubated with $4 \mu \mathrm{l}$ of $2500 \mathrm{U} / \mathrm{ml}$ Urease from Canavalia ensiformis for $20 \mathrm{~min}$ at $37^{\circ} \mathrm{C}$ in order to eliminate excess urea [19]. Subsequently, samples were mixed with $25 \mu \mathrm{l} 4 \mathrm{mM}$ ADMA substrate in PBS and incubated for $20 \mathrm{~h}$ at $37^{\circ} \mathrm{C}$. The rest of the assay was performed in a similar manner as for the lysed cells. The enzymatic activity was corrected for protein concentration.

\section{VEGF ELISA}

VEGF concentration was measured in cell culture medium, collected from confluent $3 \mathrm{~cm}$ dishes $72 \mathrm{~h}$ after seeding, and in tumour homogenates using a rat VEGF ELISA kit (PeproTech, London, UK) according to manufacturer's instructions. Values were normalized to protein concentration.

\section{Animals and tumours}

Experiments were performed in accordance with the local ethical review panel, the UK Home Office Scientific Procedures Act 1986 and the UK National Cancer Research Institute Guidelines for the Welfare and Use of Animals in Cancer Research [20]. Female (7-8 weeks old) NCr nude mice were injected subcutaneously in the flanks with $2 \times 10^{6}$ cells in $0.1 \mathrm{ml}$ PBS. Tumour volume was calculated using the ellipsoid shape formula: $(\pi / 6) \times$ Length $\times$ Width $\times$ Depth . Tumour doubling times (TDT) were calculated based on the individual tumour growth curves on a logarithmic plot using the formula: $T D T=\ln (2) /[$ slope of growth curve]. Prior to implantation, C6 DDAH cells were pre-treated for 5 days with DOX (C6 DDAH group A) or were grown in normal medium without DOX (C6 DDAH group B). Animals injected with C6 DDAH cells (groups A and B) were given drinking water containing $5 \%(\mathrm{w} / \mathrm{v})$ sucrose with or without $0.2 \mathrm{mg} / \mathrm{ml}$ DOX ( $n=6$ per group) (C6 DDAH \pm DOX group A and C6 DDAH \pm DOX group B). Additional animals $(n=4)$ were injected with constitutively DDAH I overexpressing cells (clone D27), previously engineered and characterized by Kostourou et al [5]. 


\section{Magnetic resonance imaging}

Mice bearing size-matched $\left(\sim 500 \mathrm{~mm}^{3}\right)$ tumours were anaesthetised with a $10 \mathrm{ml} / \mathrm{kg}$ intraperitoneal injection of Hypnorm $(0.315 \mathrm{mg} / \mathrm{ml}$ fentanyl citrate plus $10 \mathrm{mg} / \mathrm{ml} \mathrm{flu-}$ anisone; Janssen Pharmaceutical, Wantage, UK), Hypnovel ( $5 \mathrm{mg} / \mathrm{ml}$ midazolam; Roche, West Sussex, UK) and water (1:1:2), and positioned so the tumour hung within a threeturn 25-mm-diameter surface coil for MRI using a $4.7 \mathrm{~T}$ Varian Unity INOVA horizontal small-bore imaging system. The mouse core temperature was maintained at $37^{\circ} \mathrm{C}$ using heated air blown through the magnet bore. Blood oxygen saturation was monitored using a MouseOx Pulse Oximeter (Braintree Scientific, MA, US).

$\mathrm{T}_{2}$-weighted spin echo images were acquired from seven axial 1-mm-thick slices positioned across the whole tumour, using a repetition time (TR) of $1500 \mathrm{~ms}$, an echo time (TE) of $30 \mathrm{~ms}$, and a $128 \times 128$ matrix over a $2.56-\mathrm{cm}$ field of view. Intrinsic susceptibility MRI was performed to assess vessel function and maturation, utilizing carbogen $(95 \%$ $\mathrm{O}_{2} / 5 \% \mathrm{CO}_{2}$ ) breathing to increase blood oxygenation and localised vascular smooth muscle dilation. The changes in the tumour transverse relaxation rate $R_{2} *\left(\mathrm{~s}^{-1}\right)$ caused by perturbations in the paramagnetic deoxyhaemoglobin in the blood vessels were measured using a multi-gradient echo (MGRE) sequence. MGRE images were acquired from seven slices with TR of $450 \mathrm{~ms}$, TE of 7-56 ms, an echo spacing of $7 \mathrm{~ms}$ and flip angle $(\alpha)$ of $45^{\circ}$ during air and following a 5-min transition period during carbogen $\left(95 \% \mathrm{O}_{2} / 5 \% \mathrm{CO}_{2}\right)$ breathing [21-23].

Susceptibility contrast MRI was then performed to quantify the tumour fractional blood volume (fBV, \%). MGRE images were acquired, 5 min after air breathing was resumed, prior to and $5 \mathrm{~min}$ after intravenous injection of $5.2 \mathrm{mgFe} / \mathrm{kg}$ of the ultrasmall superparamagnetic iron oxide (USPIO) contrast agent ferumoxtran (Guerbet S.A., Villepinte, France). USPIO particles were used as a blood pool contrast agent that creates magnetic susceptibility variations close to blood vessels leading to an increase in water $R_{2} *$ in the surrounding tissue [24].

\section{MRI data analysis}

$R_{2}{ }^{*}$ maps were calculated on a voxel-by-voxel basis from MGRE image data using ImageJ and Matlab. Average apparent $R_{2}$ * relaxation rates were calculated for each slice for a region of interest (ROI), defined from the associated $\mathrm{T}_{2}$-weighted image, encompassing the whole tumour but excluding the surrounding skin and muscle. Carbogeninduced changes in $\mathrm{R}_{2}{ }^{*}\left(\Delta R_{2}{ }^{*}{ }_{\mathrm{CB}}=R_{2}{ }^{*}\right.$ carbogen $\left.-R_{2}{ }^{*}{ }_{\text {air }}\right)$ were determined over the whole tumour. Tumour fBV was determined over the same ROI from the increase in $\mathrm{R}_{2}{ }^{*}$
( $\Delta R_{2}{ }^{*}{ }_{\text {USPIO }}=R_{2}{ }^{*}$ post-USPIO $-R_{2}{ }^{*}$ pre-USPIO $)$ caused by the USPIO particles as previously described [24, 25].

\section{Histological analysis and microscopy}

Following the MRI, mice were administered intraperitoneally with $60 \mathrm{mg} / \mathrm{kg}$ of the hypoxia marker pimonidazole hydrochloride (Hypoxyprobe, Burlington, MA, USA) in PBS. After $45 \mathrm{~min}$, mice were also injected intravenously with $15 \mathrm{mg} / \mathrm{kg}$ of the perfusion marker Hoechst 33342 (Sigma-Aldrich, Dorset, UK) in PBS. Tumours were excised after $1 \mathrm{~min}$ and snap-frozen. For each tumour, three acetone-fixed cryosections $(10 \mu \mathrm{m})$ were visualized for uptake of Hoechst 33342 by fluorescence microscopy using a motorized scanning stage (Prior Scientific Instruments, Cambridge, UK) attached to a BX51 microscope (Olympus Optical, London, UK) driven by CellP (Soft Imaging System, Munster, Germany) to record composite digital images of whole tumour sections. The same sections were then processed for pimonidazole adduct formation using Hypoxyprobe-1 plus FITC-conjugated mouse monoclonal antibodies and imaged using the same stage coordinates. To assess endothelial and perivascular cell content, additional sections were stained with rat monoclonal anti-mouse CD31 antibodies [MEC 7.46] (ab7388, Abcam, Cambridge, UK), biotinylated goat anti-rat immunoglobulins (IgG) (Vector Laboratories, Peterborough, UK) and Fluorescein Avidin D (Vector Laboratories), and with rabbit polyclonal antibodies against smooth muscle actin ( $\alpha$-SMA, ab5694, Abcam) and Alexa-Fluor 546 goat anti-rabbit secondary antibodies. The cell nuclei were counterstained with DAPI (4',6-diamidino-2-phenylindole). Non-immune mouse and rabbit IgG antibodies were used in the same concentration with CD31 and $\alpha$-SMA antibodies respectively as negative isotype controls. Composite images were acquired using fluorescence microscopy as previously described [26]. Tumour sections were also stained with haematoxylin and eosin (H\&E), and composite images were acquired under light microscopy.

Post-processing was performed on composite digital images using ImageJ software [17]. ROIs of whole tumour sections were defined and fluorescent particles detected above a constant colour threshold across all sections, which was higher than background fluorescence defined from isotype control stained tumour sections. The area of the tumour section with fluorescence was determined and expressed as a percentage of the whole tumour section, and for each tumour the average across three slices was determined. ROIs were also drawn around all necrotic foci on H\&E-stained sections and necrosis was expressed as a percentage averaged across three tumour slices for each tumour. 


\section{Statistical analysis}

Data were analysed using GraphPad Prism (GraphPad Software Inc., La Jolla, CA, USA). Results are presented as mean \pm 1 standard error of the mean (SEM) with significance testing at a 5\% confidence level. Student's unpaired $t$-test was used to compare two groups and repeated measures ANOVA with Bonferroni's Multiple Comparison posttest was used to compare multiple groups.

\section{Results}

\section{NO production is limited in C6 DDAH cells}

Initial experiments confirmed that the basal production of NO by C6 parental cells was negligible and that they did not express iNOS in response to combined stimulation with cytokines and LPS (cytokine independent). As anticipated, iNOS expression was also unaffected by cytokine

a
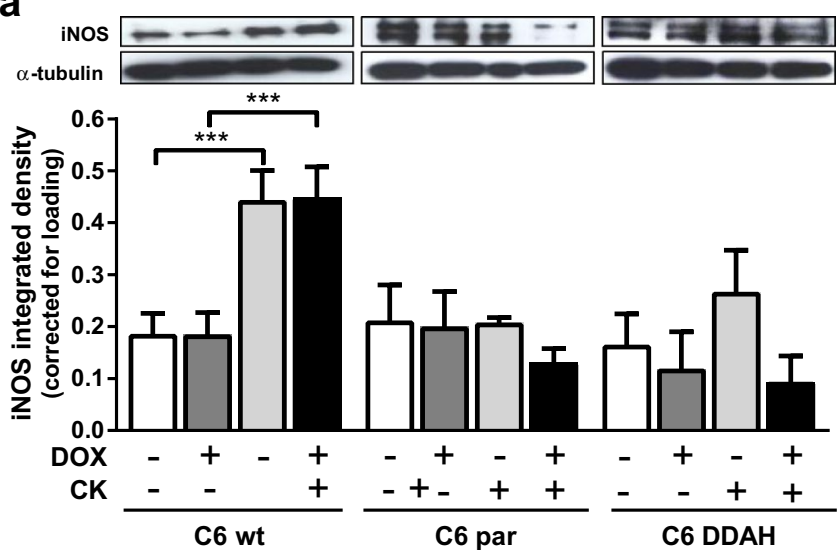

C

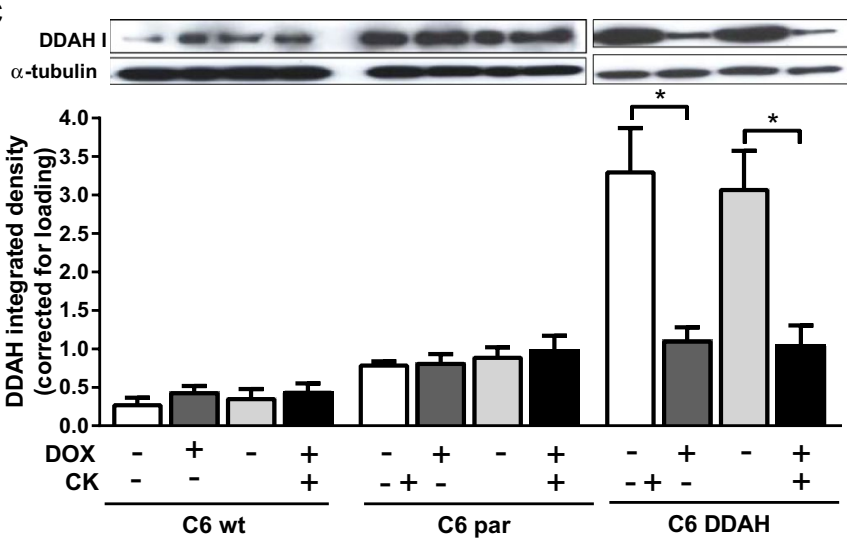

Fig. 1 In vitro characterization of C6 DDAH cells in comparison with parental cells. Cells were treated for 4 days with $2 \mu \mathrm{g} / \mathrm{ml}$ DOX. For the last $48 \mathrm{~h}$, cells were also stimulated with cytokines (10 ng/ $\mathrm{ml} \mathrm{TNF-} \alpha, 1000 \mathrm{U} / \mathrm{ml} \mathrm{IFN}-\gamma)$ and $5 \mu \mathrm{g} / \mathrm{ml}$ LPS. a iNOS (131 kDa) and c DDAH I (34 kDa) expression determined by western blot and and LPS stimulus and production of NOx was low in C6 DDAH cells, which were derived from the selected C6 parental cells. In contrast, cytokine stimulation of C6 wild-type cells led to increased iNOS protein expression (2.3-fold) and NOx production (Fig. 1a, b; Table 1). The presence of DOX did not affect iNOS expression or NO synthesis in the C6 wt, C6 par or C6 DDAH cell lines.

\section{DDAH I overexpression occurs in the absence of DOX in C6 DDAH cells}

Expression of DDAH I protein was low (endogenous expression) in C6 wild-type and C6 parental cells and expression remained unaffected by the presence of DOX (Fig. 1c; Table 1). C6 DDAH cells exhibited a tight in vitro regulation of DDAH I expression with DOX. In the absence of DOX, the cells expressed high levels of active DDAH I enzyme from the pTRE2hyg.DDAHI vector, which in turn led to high L-citrulline production. In the presence of DOX, only endogenous DDAH I was expressed, whereas removal of

\section{b}

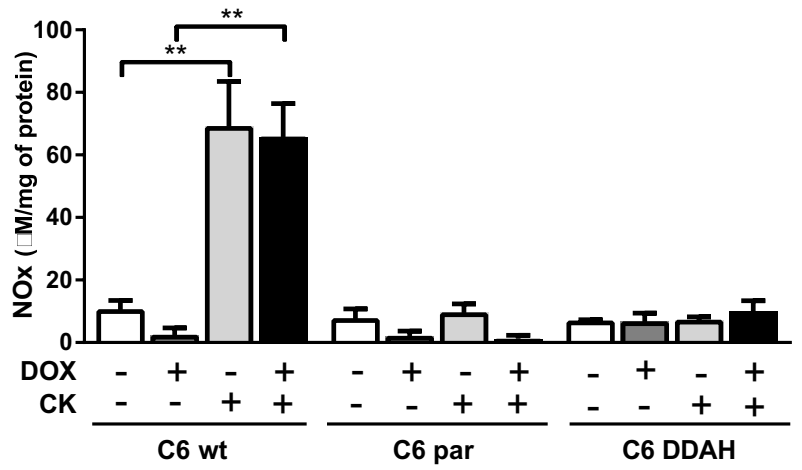

d

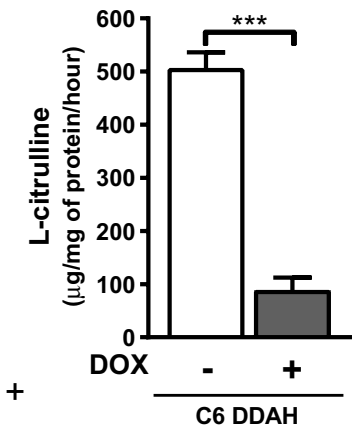

integrated density of individual bands (corrected for loading). b NOx production. d L-citrulline production evaluated after 4-h incubation with ADMA substrate. Results are mean +1 SEM of three separate experiments $(* P<0.05, * * P<0.01, * * * P<0.001)$ 
DOX led the overexpression of DDAH I, resulting in a fivefold increase in activity (Fig. 1d; Table 1). Overexpression of DDAH I did not affect the growth properties of cells in vitro; cell proliferation and death over 5 days were the same in the presence or absence of DOX (Supplementary Fig. 1a, b). Cytokines and LPS had no effect on the expression of DDAH I by all three cell lines (Fig. 1c).

\section{DDAH I up-regulation in tumours does not affect growth rate when $\mathrm{NO}$ is absent}

To investigate whether overexpression of DDAH I in tumours affects growth rate in the absence of NO, tumours derived from C6 DDAH cells were grown subcutaneously in mice and compared with constitutive DDAH I
Fig. 2 In vivo characterization of C6 DDAH tumour xenografts compared with D27 tumour xenografts. C6 DDAH cells were pre-treated with $2 \mu \mathrm{g} /$ $\mathrm{ml}$ DOX in the medium for 5 days (group A). Mice with C6 DDAH tumours were given $0.2 \mathrm{mg} / \mathrm{ml} \mathrm{DOX}$ in $5 \%(\mathrm{w} / \mathrm{v})$ sucrose or $5 \%(\mathrm{w} / \mathrm{v})$ sucrose alone ( $n=6$ per group) in the drinking water ad libitum. Mice with D27 (C6 constitutively DDAH overexpressing) tumours were given drinking water alone $(n=4)$. a, b Example western blots of tumour homogenates and mean box plot distributions of the integrated densities of the individual bands for DDAH I (a) and iNOS (b) corrected using $\alpha$-tubulin and $\alpha$-actin respectively. c L-citrulline production and (d) NOx production by tumours, determined from homogenates. e TDTs in days. Results are the mean +1 SEM of measurements made from all tumours in each group $(* P<0.05, * * P<0.01$, $* * * P<0.001, * * * * P<0.0001$, One-Way ANOVA with Bonferroni's multiple comparison post-test)

\section{a}

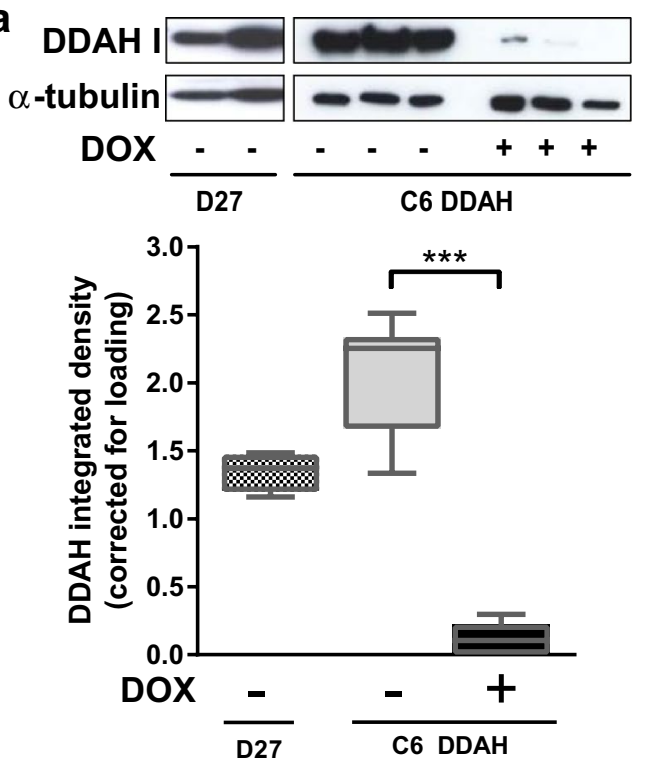

C



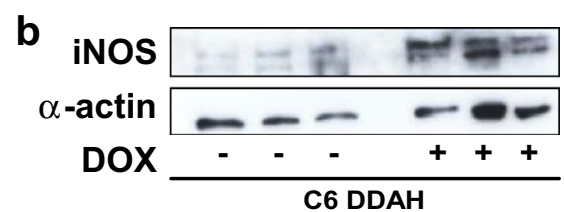

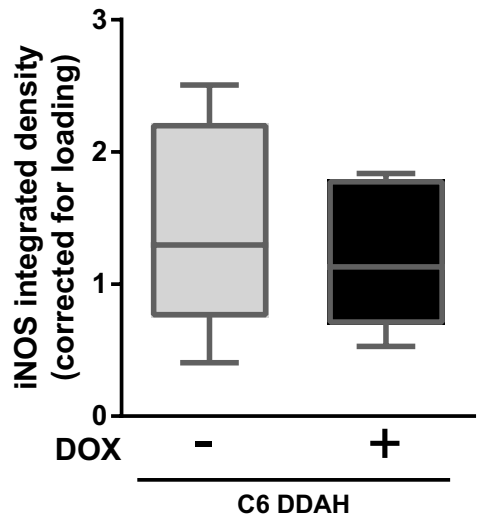

d

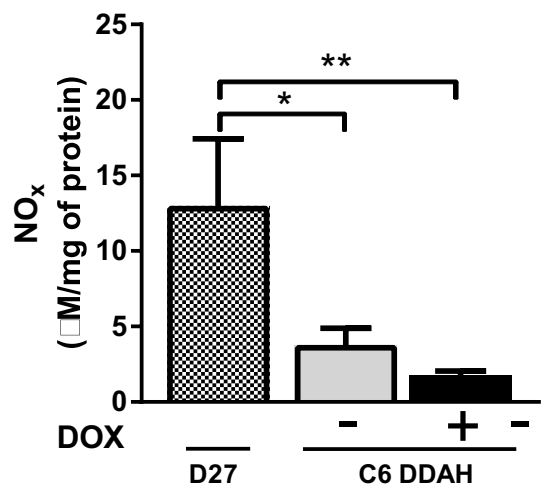

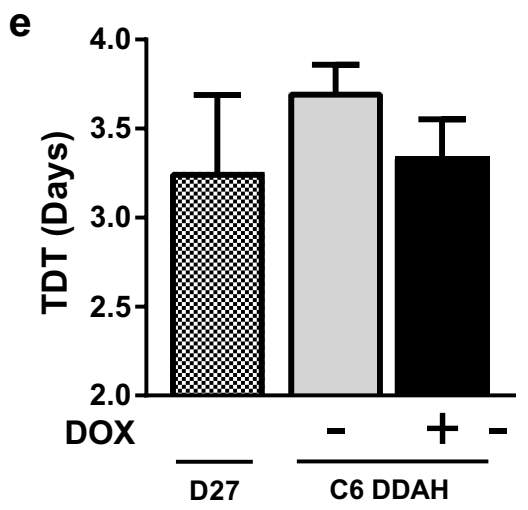


overexpressing tumours (D27), in which the DDAH/ ADMA/NO pathway is fully functional. Results from mice with C6 DDAH tumours derived from cells pretreated for 5 days with DOX (group A) are presented in Fig. 2 together with results from mice with D27 tumours. Results from mice with C6 DDAH tumours derived from cells grown in normal medium without DOX (group B) were very similar to results from group $\mathrm{A}$ and therefore are presented in Supplementary Fig. 2. DDAH I protein expression in C6 DDAH (groups A and B) tumours in mice not given DOX in the drinking water was comparable with expression in D27 tumours. However, addition of DOX in the drinking water was sufficient to "switch off" DDAH I overexpression in C6 DDAH tumours (both groups) returning to the low endogenous DDAH I expression levels (Fig. 2a, Supplementary Fig. 2a, Table 1). Production of L-citrulline, and hence DDAH enzymatic activity, was approximately five times higher in C6 DDAH tumours grown without DOX compared with those grown with DOX in both groups A and B (Fig. 2c, Supplementary Fig. 2b). Unexpectedly, D27 tumours had significantly lower levels of DDAH activity ( 18-fold) than C6 DDAH - DOX tumours (group A) (Fig. 2c). Pre-treatment of the C6 DDAH cells with DOX prior to implantation (group A) had no effect on DDAH I activity. No significant differences were observed in the expression of iNOS or the production of NOx between C6 DDAH \pm DOX tumours (group A) (Fig. 2b, d; Table 1). In contrast, NOx production was significantly higher (threefold) in D27 tumours compared with the C6 DDAH \pm DOX tumours (group
A). DDAH I overexpression in the absence of NO had no effect on the growth rate of C6 DDAH tumours (group A) (Fig. 2e, Supplementary Fig. 1c). Pre-treating the cells with or without DOX also had no effect on the growth rate of C6 DDAH tumours (groups A and B) (Fig. 2e, Supplementary Fig. 2c). All tumours grew with similar growth rates, with TDTs between 3 and 4 days.

\section{DDAH I overexpression does not alter VEGF production when NO is limited}

Having characterised the DDAH/ADMA/NO axis in the C6 DDAH cells and tumours, the effects of DDAH I overexpression on VEGF were investigated. C6 DDAH \pm DOX cells produced less VEGF than C6 wt and C6 par cells, and DDAH I overexpression in the C6 DDAH - DOX cells did not increase VEGF production (Fig. 3a; Table 1). Moreover, cytokine stimulation did not have an effect on VEGF production in C6 wt, C6 par or C6 DDAH cells in the absence or presence of DOX. Interestingly, the presence of DOX increased VEGF production by 1.8 -fold in C6 wt cells, 1.5fold in C6 par and 2.5-fold in C6 DDAH cells (Fig. 3a). In tumour homogenates, VEGF production was low in all C6 DDAH tumours (groups A and B) and no significant differences were found between tumours grown in the presence or absence of DOX (Fig. 3b, Supplementary Fig. 2d, Table 1). VEGF production was markedly higher ( 26-fold) in D27 tumours compared with C6 DDAH \pm DOX tumours (group A). Results for DDAH I expression, NO production
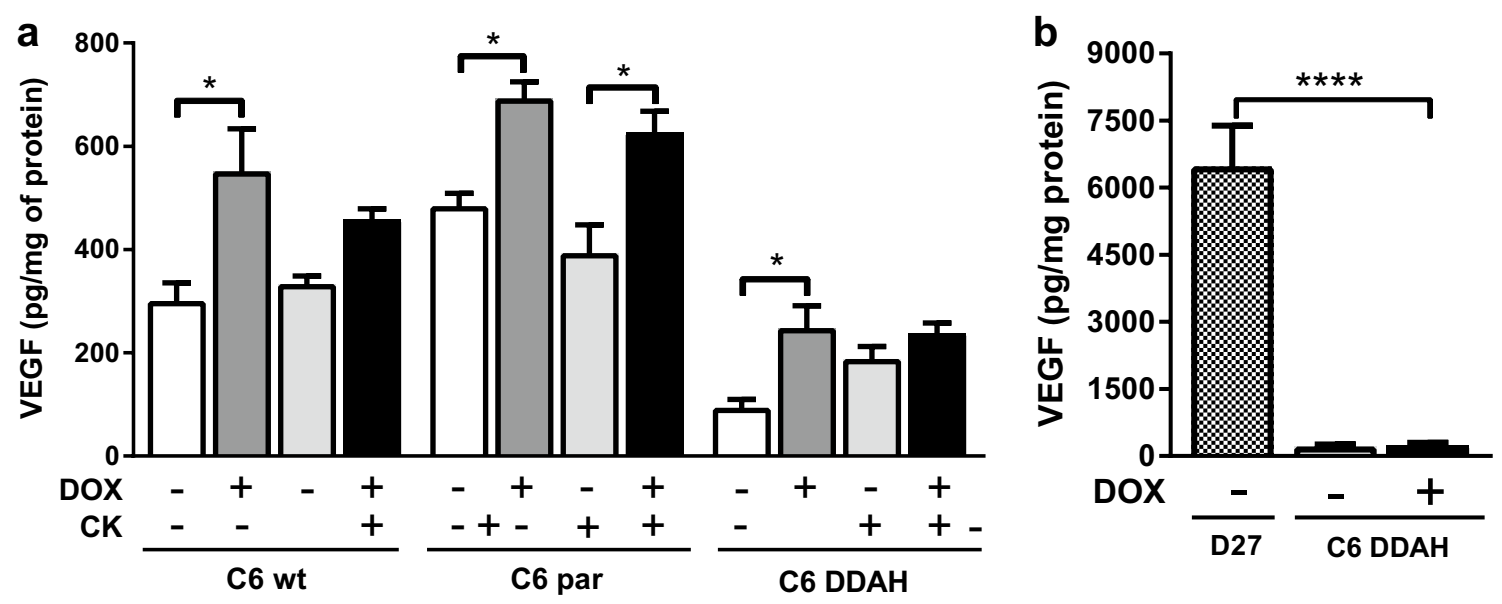

Fig. 3 Investigation of the effect of DDAH I on VEGF expression in vitro and in vivo. a VEGF levels in culture medium from cells treated with $2 \mu \mathrm{g} / \mathrm{ml}$ DOX for 6 days. For the last day, cells were also stimulated with cytokines $(10 \mathrm{ng} / \mathrm{ml}$ TNF- $\alpha, 1000 \mathrm{U} / \mathrm{ml} \mathrm{IFN-} \gamma)$ and $5 \mu \mathrm{g} / \mathrm{ml}$ LPS. Culture medium was collected from the last $24 \mathrm{~h}$ of treatment. b VEGF expression in tumour homogenates. C6 DDAH tumours (group A) derived from animals provided with drinking water containing $0.2 \mathrm{mg} / \mathrm{ml} \mathrm{DOX}$ in $5 \%(\mathrm{w} / \mathrm{v})$ sucrose or $5 \%(\mathrm{w} / \mathrm{v})$ sucrose alone ( $n=6$ per group). Mice with D27 tumours were given drinking water alone $(n=4)$. Results are mean +1 SEM of duplicate measurements from three separate in vitro experiments or from all tumours in each group $(* P<0.05, * * * * P<0.0001$, One-Way ANOVA with Bonferroni's multiple comparison post-test) 
and VEGF expression from all cell lines and tumours are briefly summarized in Table 1.

\section{In the absence of NO tumour vascular development is unaffected by DDAH I up-regulation}

Non-invasive MRI was used to explore whether DDAH I overexpression in C6 DDAH tumours (groups A and B) caused any NO-independent effects on functional tumour vasculature in vivo. Representative $T_{2}$-weighted images and parametric maps of baseline $\mathrm{R}_{2}{ }^{*}$ and carbogen-induced
$\Delta R_{2}{ }^{*}\left(\Delta R_{2}{ }^{*}{ }_{\mathrm{CB}}\right)$ from $\mathrm{D} 27$ and C6 DDAH \pm DOX tumours (group A) are shown in Fig. 4a. Parametric maps of fractional blood volume (fBV) are also shown for the C6 $\mathrm{DDAH} \pm \mathrm{DOX}$ tumours (group A). No differences in baseline $R_{2}{ }^{*}$ were found across the tumour groups (Fig. 4b, Supplementary Fig. 2e). All mice physiologically responded to carbogen breathing with an increase in blood oxygen saturation of $4 \pm 1 \%$ across all groups. However, carbogen inhalation induced a negligible change, approximately $-3.2 \mathrm{~s}^{-1}$, in mean tumour $R_{2} *$ in all cohorts (Fig. 4c, Supplementary Fig. 2f). C6 DDAH tumours typically exhibited a low fBV
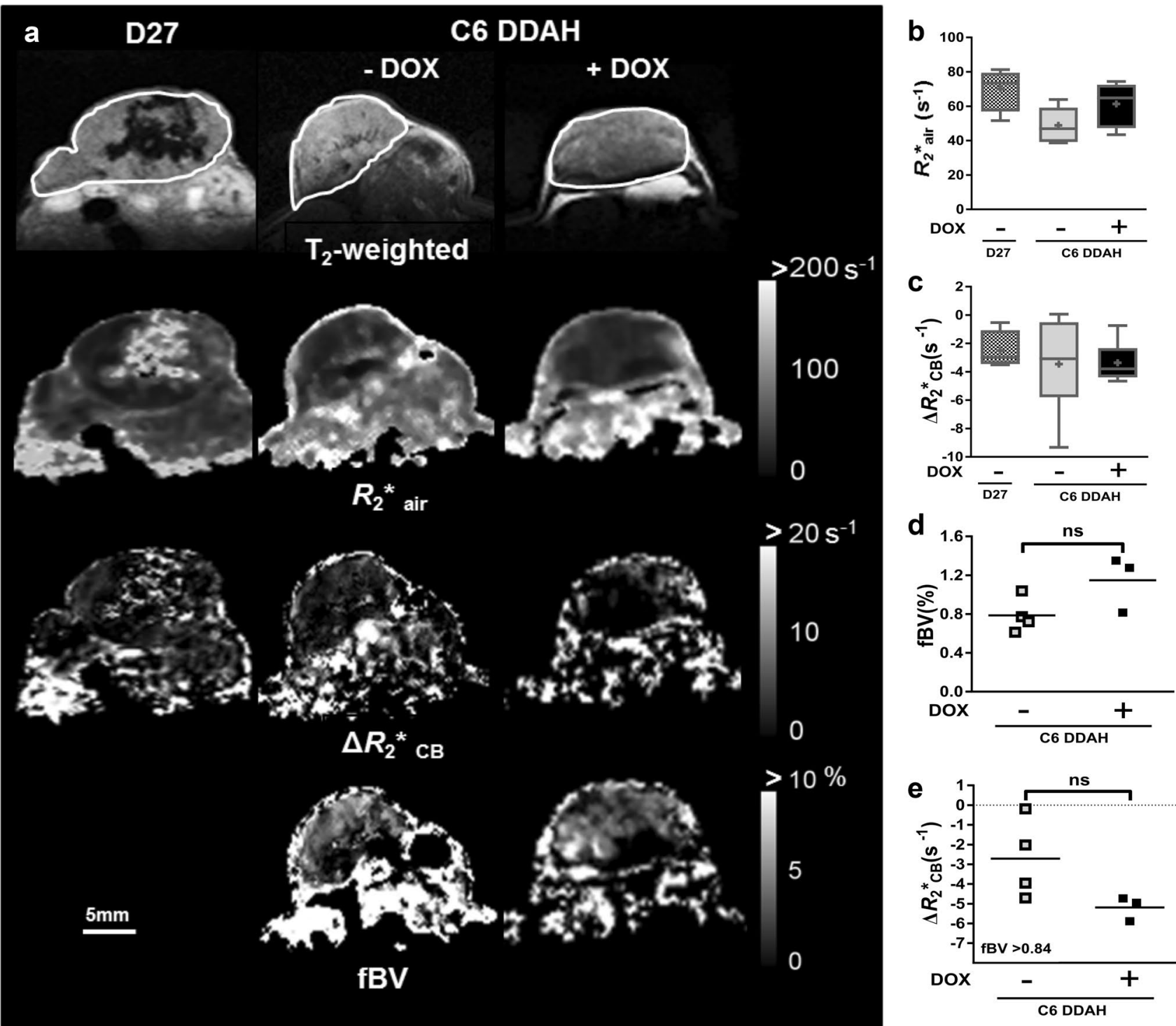

Fig. 4 The effects of DDAH I up-regulation on tumour vascular development as determined by MRI of C6 DDAH xenografts (group A). a Representative $\mathrm{T}_{2}$-weighted images, parametric maps of baseline $R_{2}{ }^{*}$ air,$\Delta R_{2}{ }^{*}{ }_{\mathrm{CB}}$ in response to carbogen and fractional blood volume (fBV) acquired from the central tumour slice. ROIs encompassing the whole tumour volume are demarcated on the $\mathrm{T}_{2}$-weighted images. Box plot distributions of the median $R_{2}{ }^{*}$ air $(\mathbf{b})$ and $\Delta R_{2}{ }^{*} \mathrm{CB}$ (c) values measured over the whole tumour volume of D27 $(n=4)$ and C6 DDAH \pm DOX $(n=6)$ xenografts. Median fBV values $(\mathbf{d})$ and median $\Delta R_{2}{ }^{*}{ }_{\mathrm{CB}}$ values (e) in regions where $\mathrm{fBV}$ is higher than the median $\mathrm{fBV}$ value of all the data of C6 DDAH - DOX $(n=4)$ and C6 $\mathrm{DDAH}+\mathrm{DOX}(n=3)$ tumours 
(less than $1.5 \%$ ), and despite a trend towards higher fBV in the C6 DDAH + DOX tumours (group A), the difference was not significant (Fig. 4d). Application of a retrospective analysis, previously described in Papaevangelou et al. [22], to the $\Delta R_{2}{ }^{*}$ Св maps, in which regions with vascular volume greater than the overall median of all the C6 DDAH tumours ( $\mathrm{fBV}>0.84 \%$ ) were selected, the $\Delta R_{2}{ }^{*}{ }_{\mathrm{CB}}$ response was smaller in C6 DDAH tumours (group A) grown without DOX compared to those grown with DOX, but this difference was not significant (Fig. 4e). No differences were observed between the baseline $R_{2}{ }^{*}$ measured during air breathing prior to carbogen challenge $\left(R_{2}{ }^{*}\right.$ air $)$ and that measured following resumption of air breathing prior to administration of USPIO particles ( $R_{2}{ }^{*}$ pre-USPIO $)$.

Ex vivo histological analysis was also used to assess the tumour vasculature. Representative histological images from D27 and C6 DDAH \pm DOX (group A) tumours are shown in Fig. 5, and some higher magnification images are shown in Supplementary Fig. 3. Hoechst was found closer to the periphery of the tumour where vessels were better perfused, whereas pimonidazole adducts were located closer to the tumour centre nearer to necrotic areas (Fig. 5a). More necrotic regions (indicated with black arrows) were observed in D27 tumours compared with C6 DDAH \pm DOX (group A) tumours (Fig. 5b).
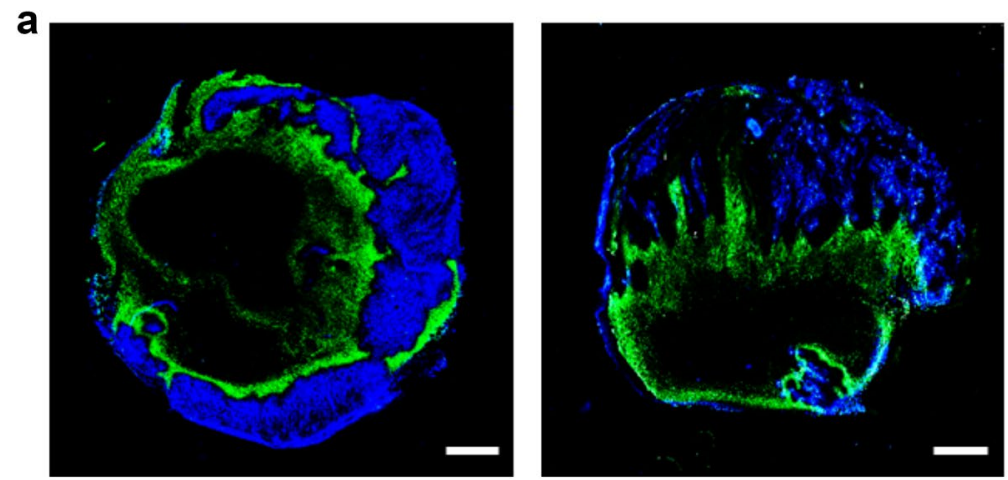
a. Hoechst \& Pimonidazole
b. H\&E
c. CD31 \& $\alpha-S M A$


C6 DDAH - DOX

C6 DDAH + DOX

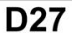

Fig. 5 Histological assessment of the vasculature of C6 $\mathrm{DDAH} \pm \mathrm{DOX}$ (group A) and D27 xenografts. a Representative composite images from frozen whole C6 DDAH \pm DOX tumour sections stained with the perfusion marker Hoechst 33342 (blue fluorescence) and the hypoxia marker pimonidazole (green fluorescence). b H\&E- stained frozen sections from C6 DDAH \pm DOX and D27 tumours indicating necrotic regions (arrows). c Composite images from frozen $\mathrm{C} 6 \mathrm{DDAH} \pm \mathrm{DOX}$ and D27 tumour sections stained with the endothelial cell marker CD31 (green fluorescence) and the perivascular cell marker $\alpha$-SMA (red fluorescence). Scale bar is $1 \mathrm{~mm}$ 



Fig.6 Quantification of histological markers of tumour vasculature for C6 DDAH \pm DOX (group A) and D27 xenografts. a Hoechst 33342 perfused area, b pimonidazole adduct area, c necrotic area, $\mathbf{d}$ endothelial cell content (CD31 stained area), e perivascular cell coverage ( $\alpha$-SMA stained area), and $\mathbf{f}$ fraction of mature vessels
$(\alpha-S M A / C D 31$ ratio) of tumour xenografts. Results are means +1 SEM of three sections per tumour for $n=4$ in the D27 group and $n=6$ in the C6 DDAH \pm DOX groups $(* P<0.05, * * P<0.01$, $* * * P<0.001)$
Mature vessel smooth muscle cells and pericytes surrounded the endothelial cell layer (Fig. 5c). White arrows in the composite images indicate large vessels where the localization of CD31 and $\alpha$-SMA in the vessel wall is more profound (Supplementary Fig. 3). DDAH I overexpression did not result in any differences in the degree of tumour perfusion (Hoechst perfused area) and hypoxia (pimonidazole adduct area) in the C6 DDAH \pm DOX tumours (group A) (Fig. 6a, b). The extent of necrosis was significantly higher in D27 tumours compared with C6 DDAH \pm DOX tumours (group A), whereas necrosis was similar in the C6 DDAH tumours (group A) independently of DDAH I expression levels (Figs. 5b, 6c). The endothelial cell content (CD31) of D27 tumours was significantly higher (3.3\%) than in C6 DDAH tumours (group A), which had an endothelial cell content of $1.7 \%$ in both the absence and presence of DDAH I expression (Figs. 5c, 6d). D27 tumours had a significantly higher perivascular cell content ( $\alpha$-SMA, $3 \%$ ) compared with C6 DDAH \pm DOX tumours (group A) $(0.6 \%)$ (Figs. 5b, 6e). The fraction of mature vessels, quantified using the ratio of $\alpha$-SMA stained area versus the CD31 stained area, was also higher in D27 tumours compared with C6 DDAH \pm DOX tumours (group A) (Fig. 6f). However, no differences were observed in the C6 DDAH tumours (group A) in the absence or presence of DOX for either CD31 or $\alpha$-SMA staining.

\section{Discussion}

In this study, a novel tetracycline-inducible DDAH I overexpression system, in which DDAH I was overexpressed in the absence of DOX, was generated from nitric 
oxide-deficient C6 parental cells, in order to investigate the direct, NO-independent role of DDAH I on tumour growth and angiogenesis. Our key findings indicate that in the absence of NO production, DDAH I overexpression had no effect on tumour growth, tumour vascular characteristics as assessed by in vivo MRI, ex vivo quantification of VEGF, vessel perfusion, hypoxia, endothelial and perivascular cell content, or necrosis.

Previous studies have shown that increased DDAH activity leads to degradation of the NOS inhibitors ADMA and L-NMMA, and therefore increases NO. This has been demonstrated in constitutive DDAH I overexpressing D27 cells and tumours, where increased DDAH I activity reduced ADMA concentration and resulted in a twofold increase in NO synthesis [5]. In our study, the DOXinducible C6 DDAH cells and tumours (groups A and B) exhibited very limited NO production independently of DOX, and therefore independently of DDAH I expression. D27 tumours produced a significantly higher concentration of NO compared with the C6 DDAH tumours ( \pm DOX). Overexpression of DDAH I in D27 tumours led to faster growth rates compared with wild-type $\mathrm{C} 6$ tumours [5]. In our study, the growth rates of the NO-deficient C6 DDAH tumours (groups A and B) were independent of DDAH I overexpression. Hence, in light of our findings, we can deduce that the effect of DDAH on D27 tumour growth reported by Kostourou et al. [5] was NO-mediated. No significant differences were observed between the DOXtreated and untreated C6 DDAH tumours in the MRI measures of $\mathrm{fBV}$ and carbogen-induced $R_{2}{ }^{*}$ changes or in the histologically assessed vessel perfusion, hypoxia, micro-vessel density and vessel maturation. This was probably due to the limited NO production in these tumours. However, in D27 tumours, where production of NO was significantly higher, perivascular cell coverage, and thus vessel maturation, were higher compared with the C6 DDAH tumours (group A). DDAH I protein expression had no effect on the resulting degree of necrosis in C6 DDAH tumours (group A), while D27 tumours, which had high NO production, were more necrotic. Previous studies have shown that high concentrations of NO can directly stimulate apoptosis or necrosis [27, 28]. In C6 DDAH tumours, in the absence of NO, altering DDAH I expression in order to regulate ADMA did not have an effect on the vascular growth of those tumours. We can therefore conclude that DDAH I overexpression alone was not sufficient to overcome the effects of low NO production on tumour growth and vascular development. This is in agreement with a previous study showing that antisense iNOS expressing and DDAH I overexpressing C6 tumours (referred to as ASD10) had similar growth rates and vessel perfusion compared with AS7 tumours (antisense iNOS expressing C6 tumours), and slower growth rates and lower perfusion than D27 (DDAH I overexpressing) and wild-type C6 tumours [29].

The DDAH I/ADMA pathway is known to regulate VEGF-induced angiogenesis in an NO-dependent manner $[30,31]$. In the present study, VEGF expression was significantly greater in D27 tumours compared with C6 DDAH tumours (groups A and B) and this correlated with increased NO production in D27 tumours. Increased VEGF expression in D27 tumours (DDAH I overexpressing C6 tumours) has previously been reported in vitro and in vivo [5]. This increased VEGF expression, downstream of an increase in NO production, was diminished in C6 tumours overexpressing a mutant inactive DDAH I enzyme [32]. However, ADMA and DDAH can also regulate VEGF expression via NO-independent mechanisms, such as via DDAH binding to protein kinase A (PKA) and subsequent phosphorylation of the transcription factor Sp1 [10]. In our study, DDAH I overexpression in C6 DDAH cells and tumours and NO accumulation in wild-type $\mathrm{C} 6$ cells did not have an effect on VEGF expression, suggesting that in C6 tumours VEGF production is not reliant only on the production of NO. This finding was supported by the fact that in both C6 DDAH and C6 parental cells VEGF was expressed despite the limited NO concentration. Yang et al. [33] suggested that in C6 cells VEGF expression was adjusted and maintained at a certain level according to factors, such as MMPs, HIF-1 and $\mathrm{pH}$. Overall, our study and previous studies suggest that C6 cells can exert both NO-dependent and NO-independent regulation of VEGF.

DOX led to an in vitro increase in VEGF in all cell lines in this study in the absence of cytokine stimulus. However, the effect was diminished in the presence of cytokine and LPS stimulus. Moreover, no similar effect of DOX on VEGF production was observed in the tumour microenvironment in the in vivo model. There are studies that have reported both positive and negative effects of DOX on VEGF and angiogenesis; however, the mechanism of this interaction still remains elusive. A study in murine melanoma tumours has shown that doxycycline treatment increased VEGF expression in vivo, possibly due to aggravation of hypoxia [34]. On the contrary, Su et al. have shown that doxycycline inhibits VEGF-induced angiogenesis in vivo [35]. In our study, the effect of DOX on VEGF does not impinge on our major findings, since this effect was only observed in vitro not in vivo and mainly in the absence of cytokine and LPS stimulus.

We observed that DDAH I activity was significantly lower in D27 tumours compared with C6 DDAH - DOX tumours (groups A and B), despite them having similar levels of DDAH I expression. Leiper et al. [36] have shown that in situations of oxidative or nitrosative stress, NO can inhibit bacterial DDAH enzymatic activity by reversible $S$-nitrosylation of the active-site cysteine (Cys249) of the Cys-His-Glu catalytic triad. This provides a homeostatic 
mechanism to regulate NO; increased $\mathrm{NO}$ production reduces DDAH activity, which in turn leads to ADMA accumulation and inhibition of NOS, thereby restraining further NO production [36]. Therefore, the observations in the present study that D27 tumours showed (a) reduced DDAH activity, and (b) increased NO production could be explained by this negative feedback loop in which NO controls DDAH activity. Kostourou et al. [5] have shown that DDAH activity, assessed by conversion of ${ }^{14} \mathrm{C}$-labelled L-NMMA to ${ }^{14} \mathrm{C}$-labelled citrulline, was high in D27 tumours, suggesting that the time point during tumour growth at which DDAH activity is measured is of great importance. Thus, another explanation for the reduced DDAH activity in the D27 cells observed in the present study could be that at the time of tumour excision (the time point at which DDAH activity was assessed), the amount of DDAH enzyme required to metabolize all the available ADMA was being used, whereas the remainder unused enzyme became inactivated as it was not required.

We have now shown that in C6 gliomas in the absence of NO, endogenous DDAH I overexpression did not alter tumour growth or vascular development. Our data reinforce the hypothesis that regulating DDAH I expression in gliomas by using a DDAH inhibitor could be a useful anti-cancer therapeutic approach, as it could lead to indirect anti-tumour and anti-angiogenic effects by raising endogenous levels of ADMA leading to a subsequent inhibition of NO synthesis. PD 404182, an irreversible inhibitor of human DDAH I, has previously shown therapeutic potential in septic shock-induced vascular collapse. PD 404182 led to ADMA-mediated reduction of NO and abrogated the formation of tube-like structures by endothelial cells in an in vitro angiogenesis assay [37]. Another potent inhibitor of human DDAH I, Cl-NIO, has been shown to decrease NO production in A375 melanoma cells [38]. Such inhibitors appear to be promising tools for the control of methylarginine-mediated $\mathrm{NO}$ and could be used as antiangiogenic therapeutic compounds in diseases where $\mathrm{NO}$ production is elevated, such as septic shock, or in diseases characterized by pathologically excessive angiogenesis, such as different types of cancer. Our study provides a rationale for developing novel DDAH inhibitors, such as arginine analogue 10a [39], to control NO biosynthesis and target tumour angiogenesis.

Acknowledgements This work was supported by a Medical Research Council and St. George's Hospital Medical School PhD studentship awarded to EP (Grant No. G0700019/G0800110), the Association for International Cancer Research (Grant No. 09-0310) and the Biotechnology and Biological Sciences Research Council (Grant No. S20430). The authors would like to thank Prof Michal Neeman for providing the C6 parental cells, and Glyn Fisher and his staff for animal care.
Funding This study was funded by a Medical Research Council and St. George's Hospital Medical School PhD studentship awarded to EP (Grant No. G0700019/G0800110), the Association for International Cancer Research (Grant No. 09-0310) and the Biotechnology and Biological Sciences Research Council (Grant No. S20430).

\section{Compliance with ethical standards}

Conflict of interest The authors declare that they have no conflict of interest.

Ethical approval All applicable international, national, and/or institutional guidelines for the care and use of animals were followed. All procedures performed in studies involving animals were in accordance with the ethical standards of the institution at which the studies were conducted. This article does not contain any studies with human participants performed by any of the authors.

Open Access This article is distributed under the terms of the Creative Commons Attribution 4.0 International License (http://creativeco mmons.org/licenses/by/4.0/), which permits unrestricted use, distribution, and reproduction in any medium, provided you give appropriate credit to the original author(s) and the source, provide a link to the Creative Commons license, and indicate if changes were made.

\section{References}

1. Burke AJ, Sullivan FJ, Giles FJ, Glynn SA (2013) The yin and yang of nitric oxide in cancer progression. Carcinogenesis 34(3):503-512. https://doi.org/10.1093/carcin/bgt034

2. MacAllister RJ, Parry H, Kimoto M, Ogawa T, Russell RJ, Hodson H, Whitley GS, Vallance P (1996) Regulation of nitric oxide synthesis by dimethylarginine dimethylaminohydrolase. Br J Pharmacol 119(8):1533-1540

3. Ogawa T, Kimoto M, Sasaoka K (1989) Purification and properties of a new enzyme, NG, NG-dimethylarginine dimethylaminohydrolase, from rat kidney. J Biol Chem 264(17):10205-10209

4. Leiper JM, Santa Maria J, Chubb A, MacAllister RJ, Charles IG, Whitley GS, Vallance P (1999) Identification of two human dimethylarginine dimethylaminohydrolases with distinct tissue distributions and homology with microbial arginine deiminases. Biochem J 343(Pt 1):209-214

5. Kostourou V, Robinson SP, Cartwright JE, Whitley GS (2002) Dimethylarginine dimethylaminohydrolase I enhances tumour growth and angiogenesis. Br J Cancer 87(6):673-680. https:// doi.org/10.1038/sj.bjc. 6600518

6. Hulin JA, Tommasi S, Elliot D, Hu DG, Lewis BC, Mangoni AA (2017) MiR-193b regulates breast cancer cell migration and vasculogenic mimicry by targeting dimethylarginine dimethylaminohydrolase 1. Sci Rep 7(1):13996. https://doi.org/10.1038/s4159 8-017-14454-1

7. Kostourou V, Robinson SP, Whitley GS, Griffiths JR (2003) Effects of overexpression of dimethylarginine dimethylaminohydrolase on tumor angiogenesis assessed by susceptibility magnetic resonance imaging. Cancer Res 63(16):4960-4966

8. Kostourou V, Troy H, Murray JF, Cullis ER, Whitley GS, Griffiths JR, Robinson SP (2004) Overexpression of dimethylarginine dimethylaminohydrolase enhances tumor hypoxia: an insight into the relationship of hypoxia and angiogenesis in vivo. Neoplasia 6(4):401-411. https://doi.org/10.1593/neo.04109 
9. Leiper J, Nandi M (2011) The therapeutic potential of targeting endogenous inhibitors of nitric oxide synthesis. Nat Rev Drug Discov 10(4):277-291. https://doi.org/10.1038/nrd3358

10. Hasegawa K, Wakino S, Tanaka T, Kimoto M, Tatematsu S, Kanda T, Yoshioka K, Homma K, Sugano N, Kurabayashi M, Saruta T, Hayashi K (2006) Dimethylarginine dimethylaminohydrolase 2 increases vascular endothelial growth factor expression through Sp1 transcription factor in endothelial cells. Arterioscler Thromb Vasc Biol 26(7):1488-1494. https://doi.org/10.1161/01. ATV.0000219615.88323.b4

11. Smith CL, Birdsey GM, Anthony S, Arrigoni FI, Leiper JM, Vallance P (2003) Dimethylarginine dimethylaminohydrolase activity modulates ADMA levels, VEGF expression, and cell phenotype. Biochem Biophys Res Commun 308(4):984-989

12. Mocellin S, Bronte V, Nitti D (2007) Nitric oxide, a double edged sword in cancer biology: searching for therapeutic opportunities. Med Res Rev 27(3):317-352. https://doi.org/10.1002/med.20092

13. Ridnour LA, Thomas DD, Donzelli S, Espey MG, Roberts DD, Wink DA, Isenberg JS (2006) The biphasic nature of nitric oxide responses in tumor biology. Antioxid Redox Signal 8(7-8):13291337. https://doi.org/10.1089/ars.2006.8.1329

14. Miranda KM, Espey MG, Wink DA (2001) A rapid, simple spectrophotometric method for simultaneous detection of nitrate and nitrite. Nitric Oxide 5(1):62-71. https://doi.org/10.1006/ niox.2000.0319

15. Di Rosa M, Radomski M, Carnuccio R, Moncada S (1990) Glucocorticoids inhibit the induction of nitric oxide synthase in macrophages. Biochem Biophys Res Commun 172(3):1246-1252

16. Bradford MM (1976) A rapid and sensitive method for the quantitation of microgram quantities of protein utilizing the principle of protein-dye binding. Anal Biochem 72:248-254

17. Schneider CA, Rasband WS, Eliceiri KW (2012) NIH Image to ImageJ: 25 years of image analysis. Nat Methods 9(7):671-675

18. Knipp M, Vasak M (2000) A colorimetric 96-well microtiter plate assay for the determination of enzymatically formed citrulline. Anal Biochem 286(2):257-264. https://doi.org/10.1006/ abio.2000.4805

19. Tain YL, Baylis C (2007) Determination of dimethylarginine dimethylaminohydrolase activity in the kidney. Kidney Int 72(7):886-889

20. Workman P, Aboagye EO, Balkwill F, Balmain A, Bruder G, Chaplin DJ, Double JA, Everitt J, Farningham DA, Glennie MJ, Kelland LR, Robinson V, Stratford IJ, Tozer GM, Watson S, Wedge SR, Eccles SA, Committee of the National Cancer Research I (2010) Guidelines for the welfare and use of animals in cancer research. Br J Cancer 102(11):1555-1577. https://doi. org/10.1038/sj.bjc.6605642

21. Howe FA, Robinson SP, McIntyre DJ, Stubbs M, Griffiths JR (2001) Issues in flow and oxygenation dependent contrast (FLOOD) imaging of tumours. NMR Biomed 14(7-8):497-506

22. Papaevangelou E, Whitley GS, Johnstone AP, Robinson SP, Howe FA (2016) Investigating the role of tumour cell derived iNOS on tumour growth and vasculature in vivo using a tetracycline regulated expression system. Int J Cancer 138(11):2678-2687. https ://doi.org/10.1002/ijc.29997

23. Robinson SP, Rodrigues LM, Howe FA, Stubbs M, Griffiths JR (2001) Effects of different levels of hypercapnic hyperoxia on tumour R(2)* and arterial blood gases. Magn Reson Imaging 19(2):161-166

24. Tropres I, Grimault S, Vaeth A, Grillon E, Julien C, Payen JF, Lamalle L, Decorps M (2001) Vessel size imaging. Magn Reson Med 45(3):397-408

25. Howe FA, McPhail LD, Griffiths JR, McIntyre DJ, Robinson SP (2008) Vessel size index magnetic resonance imaging to monitor the effect of antivascular treatment in a rodent tumor model. Int J Radiat Oncol Biol Phys 71(5):1470-1476. https://doi. org/10.1016/j.ijrobp.2008.04.027
26. Papaevangelou E, Almeida GS, Jamin Y, Robinson SP, deSouza NM (2015) Diffusion-weighted MRI for imaging cell death after cytotoxic or apoptosis-inducing therapy. Br J Cancer 112(9):1471-1479. https://doi.org/10.1038/bjc.2015.134

27. Bonfoco E, Krainc D, Ankarcrona M, Nicotera P, Lipton SA (1995) Apoptosis and necrosis: two distinct events induced, respectively, by mild and intense insults with $N$-methyl-D-aspartate or nitric oxide/superoxide in cortical cell cultures. Proc Natl Acad Sci USA 92(16):7162-7166

28. Virag L, Scott GS, Cuzzocrea S, Marmer D, Salzman AL, Szabo C (1998) Peroxynitrite-induced thymocyte apoptosis: the role of caspases and poly (ADP-ribose) synthetase (PARS) activation. Immunology 94(3):345-355

29. Kostourou V (2002) The role of nitric oxide and dimethylarginine dimethylaminohydrolase in tumour growth and angiogenesis. Doctoral thesis, St. George's Hospital Medical School, University of London

30. Fiedler LR, Bachetti T, Leiper J, Zachary I, Chen L, Renne T, Wojciak-Stothard B (2009) The ADMA/DDAH pathway regulates VEGF-mediated angiogenesis. Arterioscler Thromb Vasc Biol 29(12):2117-2124. https://doi.org/10.1161/ATVBA HA.109.194035

31. Buijs N, Oosterink JE, Jessup M, Schierbeek H, Stolz DB, Houdijk AP, Geller DA, van Leeuwen PA (2017) A new key player in VEGF-dependent angiogenesis in human hepatocellular carcinoma: dimethylarginine dimethylaminohydrolase 1 . Angiogenesis. https://doi.org/10.1007/s10456-017-9567-4

32. Boult JK, Walker-Samuel S, Jamin Y, Leiper JM, Whitley GS, Robinson SP (2011) Active site mutant dimethylarginine dimethylaminohydrolase 1 expression confers an intermediate tumour phenotype in C6 gliomas. J Pathol 225(3):344-352. https://doi. org/10.1002/path.2904

33. Yang L, Lin Z, Huang Q, Lin J, Chen Z, Zhou L, Zhang P (2011) Effect of vascular endothelial growth factor on remodeling of C6 glioma tissue in vivo. J Neurooncol 103(1):33-41. https://doi. org/10.1007/s11060-010-0356-9

34. Sun B, Zhang S, Zhang D, Yin X, Wang S, Gu Y, Wang Y (2007) Doxycycline influences microcirculation patterns in B16 melanoma. Exp Biol Med (Maywood) 232(10):1300-1307. https://doi. org/10.3181/0705-RM-145

35. Su W, Li Z, Li F, Chen X, Wan Q, Liang D (2013) Doxycyclinemediated inhibition of corneal angiogenesis: an MMP-independent mechanism. Invest Ophthalmol Vis Sci 54(1):783-788. https ://doi.org/10.1167/iovs.12-10323

36. Leiper J, Murray-Rust J, McDonald N, Vallance P (2002) S-nitrosylation of dimethylarginine dimethylaminohydrolase regulates enzyme activity: further interactions between nitric oxide synthase and dimethylarginine dimethylaminohydrolase. Proc Natl Acad Sci USA 99(21):13527-13532. https://doi.org/10.1073/ pnas.212269799

37. Ghebremariam YT, Erlanson DA, Cooke JP (2014) A novel and potent inhibitor of dimethylarginine dimethylaminohydrolase: a modulator of cardiovascular nitric oxide. J Pharmacol Exp Ther 348(1):69-76. https://doi.org/10.1124/jpet.113.206847

38. Wang Y, Hu S, Gabisi AM Jr, Er JA, Pope A, Burstein G, Schardon CL, Cardounel AJ, Ekmekcioglu S, Fast W (2014) Developing an irreversible inhibitor of human DDAH-1, an enzyme upregulated in melanoma. ChemMedChem 9(4):792-797. https ://doi.org/10.1002/cmdc.201300557

39. Tommasi S, Zanato C, Lewis BC, Nair PC, Dall'Angelo S, Zanda M, Mangoni AA (2015) Arginine analogues incorporating carboxylate bioisosteric functions are micromolar inhibitors of human recombinant DDAH-1. Org Biomol Chem 13(46):11315-11330. https://doi.org/10.1039/c5ob01843a 